\title{
Orthogonal stability of an additive-quartic functional equation with the fixed point alternative
}

\author{
Sung Jin Lee ${ }^{1}$, Choonkil Park ${ }^{2}$ and Reza Saadati ${ }^{3^{*}}$
}

\footnotetext{
* Correspondence: rsaadati@eml.cc ${ }^{3}$ Department of Mathematics and Computer Science, Iran University of Science and Technology, Behshahr, Iran

Full list of author information is available at the end of the article
}

\begin{abstract}
Using the fixed point method, we prove the Hyers-Ulam stability of the orthogonally additive-quartic functional equation

$$
\begin{aligned}
f(2 x+y)+f(2 x-y) & =4 f(x+y)+4 f(x-y) \\
& +10 f(x)+14 f(-x)-3 f(y)-3 f(-y)
\end{aligned}
$$

for all $x, y$ with $x \perp y$, where $\perp$ is the orthogonality in the sense of Rätz.

AMS Subject Classification: Primary, 39B55; 47H10; 39B52; 46H25.

Keywords: Hyers-Ulam stability, orthogonally additive-quartic functional equation, fixed point, orthogonality space
\end{abstract}

\section{Introduction and preliminaries}

Assume that $X$ is a real inner product space and $f: X \rightarrow \mathcal{R}$ is a solution of the orthogonal Cauchy functional equation $f(x+y)=f(x)+f(y),\langle x, y\rangle=0$. By the Pythagorean theorem $f(x)=\|x\|^{2}$ is a solution of the conditional equation. Of course, this function does not satisfy the additivity equation everywhere. Thus orthogonal Cauchy equation is not equivalent to the classic Cauchy equation on the whole inner product space.

Pinsker [1] characterized orthogonally additive functionals on an inner product space when the orthogonality is the ordinary one in such spaces. Sundaresan [2] generalized this result to arbitrary Banach spaces equipped with the Birkhoff-James orthogonality. The orthogonal Cauchy functional equation

$$
f(x+y)=f(x)+f(y), \quad x \perp y,
$$

in which $\perp$ is an abstract orthogonality relation, was first investigated by Gudder and Strawther [3]. They defined $\perp$ by a system consisting of five axioms and described the general semi-continuous real-valued solution of conditional Cauchy functional equation. In 1985, Rätz [4] introduced a new definition of orthogonality by using more restrictive axioms than of Gudder and Strawther. Moreover, he investigated the structure of orthogonally additive mappings. Rätz and Szabó [5] investigated the problem in a rather more general framework.

Let us recall the orthogonality in the sense of Rätz [4].

(c) 2012 Lee et al; licensee Springer. This is an Open Access article distributed under the terms of the Creative Commons Attribution License (http://creativecommons.org/licenses/by/2.0), which permits unrestricted use, distribution, and reproduction in any medium, provided the original work is properly cited. 
Suppose $X$ is a real vector space with $\operatorname{dim} X \geq 2$ and $\perp$ is a binary relation on $X$ with the following properties:

$\left(O_{1}\right)$ totality of $\perp$ for zero: $x \perp 0,0 \perp x$ for all $x \in X$;

$\left(O_{2}\right)$ independence: if $x, y \in X-\{0\}, x \perp y$, then $x, y$ are linearly independent;

$\left(O_{3}\right)$ homogeneity: if $x, y \in X, x \perp y$, then $\alpha x \perp \beta y$ for all $\alpha, \beta \in \mathcal{R}$;

$\left(O_{4}\right)$ the Thalesian property: if $P$ is a 2-dimensional subspace of $X, x \in P$ and $\lambda \in \mathcal{R}_{+}$, which is the set of nonnegative real numbers, then there exists $y_{0} \in P$ such that $x \perp y_{0}$ and $x+y_{0} \perp \lambda x-y_{0}$.

The pair $(X, \perp)$ is called an orthogonality space. By an orthogonality normed space we mean an orthogonality space having a normed structure.

Some interesting examples are

(i) The trivial orthogonality on a vector space $X$ defined by $\left(O_{1}\right)$, and for non-zero elements $x, y \in X, x \perp y$ if and only if $x, y$ are linearly independent.

(ii) The ordinary orthogonality on an inner product space $(X,\langle.,\rangle$.$) given by x \perp y$ if and only if $\langle x, y\rangle=0$.

(iii) The Birkhoff-James orthogonality on a normed space $(X,\|\|$.$) defined by x \perp y$ if and only if $\|x+\lambda y\| \geq\|x\|$ for all $\lambda \in \mathcal{R}$.

The relation $\perp$ is called symmetric if $x \perp y$ implies that $y \perp x$ for all $x, y \in X$. Clearly examples (i) and (ii) are symmetric but example (iii) is not. It is remarkable to note, however, that a real normed space of dimension greater than 2 is an inner product space if and only if the Birkhoff-James orthogonality is symmetric. There are several orthogonality notions on a real normed space such as Birkhoff-James, Boussouis, Singer, Carlsson, unitary-Boussouis, Roberts, Phythagorean, isosceles and Diminnie (see [6-11]).

The stability problem of functional equations originated from the following question of Ulam [12]: Under what condition does there exist an additive mapping near an approximately additive mapping? In 1941, Hyers [13] gave a partial affirmative answer to the question of Ulam in the context of Banach spaces. In 1978, Rassias [14] extended the theorem of Hyers by considering the unbounded Cauchy difference $\| f(x+y)-f(x)-f$ $(y) \| \leq \varepsilon\left(\|x\|^{p}+\|y\|^{p}\right),(\varepsilon>0, p \in[0,1))$. The result of Rassias has provided a lot of influence in the development of what we now call generalized Hyers-Ulam stability or HyersUlam stability of functional equations. During the last decades several stability problems of functional equations have been investigated in the spirit of Hyers-Ulam-Rassias. The reader is referred to [15-18] and references therein for detailed information on stability of functional equations.

Ger and Sikorska [19] investigated the orthogonal stability of the Cauchy functional equation $f(x+y)=f(x)+f(y)$, namely, they showed that if $f$ is a mapping from an orthogonality space $X$ into a real Banach space $Y$ and $\|f(x+y)-f(x)-f(y)\| \leq \varepsilon$ for all $x, y \in X$ with $x \perp y$ and some $\varepsilon>0$, then there exists exactly one orthogonally additive mapping $g: X \rightarrow Y$ such that $\|f(x)-g(x)\| \leq \frac{16}{3} \varepsilon$ for all $x \in X$.

The first author treating the stability of the quadratic equation was Skof [20] by proving that if $f$ is a mapping from a normed space $X$ into a Banach space $Y$ satisfying $\|f(x+y)+f(x-y)-2 f(x)-2 f(y)\| \leq \varepsilon$ for some $\varepsilon>0$, then there is a unique quadratic mapping $g: X \rightarrow Y$ such that $\|f(x)-g(x)\| \leq \frac{\varepsilon}{2}$. Cholewa [21] extended the Skof's theorem by replacing $X$ by an abelian group $G$. The Skof's result was later generalized by 
Czerwik [22] in the spirit of Hyers-Ulam-Rassias. The stability problem of functional equations has been extensively investigated by some mathematicians (see [23-27]).

The orthogonally quadratic equation

$$
f(x+y)+f(x-y)=2 f(x)+2 f(y), \quad x \perp y
$$

was first investigated by Vajzović [28] when $X$ is a Hilbert space, $Y$ is the scalar field, $f$ is continuous and $\perp$ means the Hilbert space orthogonality. Later, Drljević [29], Fochi [30], Moslehian [31,32] and Szabó [33] generalized this result. See also [34,35].

Let $X$ be a set. A function $d: X \times X \rightarrow[0, \infty]$ is called a generalized metric on $X$ if $d$ satisfies

(1) $d(x, y)=0$ if and only if $x=y$;

(2) $d(x, y)=d(y, x)$ for all $x, y \in X$;

(3) $d(x, z) \leq d(x, y)+d(y, z)$ for all $x, y, z \in X$.

We recall a fundamental result in fixed point theory.

Theorem 1.1 [36,37]Let $(X, d)$ be a complete generalized metric space and let $J: X \rightarrow X$ be a strictly contractive mapping with Lipschitz constant $\alpha<1$. Then for each given element $x \in X$, either

$$
d\left(J^{n} x, J^{n+1} x\right)=\infty
$$

for all nonnegative integers $n$ or there exists a positive integer $n_{0}$ such that

(1) $d\left(J^{n} x, J^{n+1} x\right)<\infty, \forall n \geq n_{0}$;

(2) the sequence $\left\{f^{n} x\right\}$ converges to a fixed point $y^{*}$ of $J$;

(3) $y^{*}$ is the unique fixed point of $J$ in the set $Y=\left\{y \in X \mid d\left(J^{n_{0}} x, y\right)<\infty\right\}$;

(4) $d(y, y *) \leq \frac{1}{1-\alpha} d(y$, Jy) for all $y \in Y$.

In 1996, Isac and Rassias [38] were the first to provide applications of stability theory of functional equations for the proof of new fixed point theorems with applications. By using fixed point methods, the stability problems of several functional equations have been extensively investigated by a number of authors (see [39-45]).

In [46], Lee et al. considered the following quartic functional equation

$$
f(2 x+y)+f(2 x-y)=4 f(x+y)+4 f(x-y)+24 f(x)-6 f(y) .
$$

It is easy to show that the function $f(x)=x^{4}$ satisfies the functional equation (1), which is called a quartic functional equation and every solution of the quartic functional equation is said to be a quartic mapping.

This paper is organized as follows: In Section 2, we prove the Hyers-Ulam stability of the orthogonally additive-quartic functional equation

$$
\begin{aligned}
f(2 x+y)+f(2 x-y) & =4 f(x+y)+4 f(x-y) \\
& +10 f(x)+14 f(-x)-3 f(y)-3 f(-y)
\end{aligned}
$$

in orthogonality spaces for an odd mapping. 
In Section 3, we prove the Hyers-Ulam stability of the orthogonally additive-quartic functional equation (2) in orthogonality spaces for an even mapping.

Throughout this paper, assume that $(X, \perp)$ is an orthogonality space and that $\left(Y,\|\cdot\|_{Y}\right)$ is a real Banach space.

\section{Stability of the orthogonally additive-quadratic functional equation: an odd mapping case}

In this section, applying some ideas from $[16,19]$, we deal with the stability problem for the orthogonally additive-quadratic functional equation

$$
\begin{aligned}
D f(x, y): & =f(2 x+y)+f(2 x-y)-4 f(x+y)-4 f(x-y) \\
& -10 f(x)-14 f(-x)+3 f(y)+3 f(-y)
\end{aligned}
$$

for all $x, y \in X$ with $x \perp y$ : an odd mapping case.

Definition 2.1 A mapping $f: X \rightarrow Y$ is called an orthogonally additive mapping if

$$
f(2 x+y)+f(2 x-y)=4 f(x+y)+4 f(x-y)-4 f(x)
$$

for all $x, y \in X$ with $x \perp y$.

Theorem 2.2 Let $\phi: X^{2} \rightarrow[0, \infty)$ be a function such that there exists an $\alpha<1$ with

$$
\varphi(x, y) \leq 2 \alpha \varphi\left(\frac{x}{2}, \frac{y}{2}\right)
$$

for all $x, y \in X$ with $x \perp y$. Let $f: X \rightarrow Y$ be an odd mapping satisfying

$$
\|D f(x, y)\|_{Y} \leq \varphi(x, y)
$$

for all $x, y \in X$ with $x \perp y$. Then there exists a unique orthogonally additive mapping $L: X \rightarrow Y$ such that

$$
\|f(x)-L(x)\|_{Y} \leq \frac{1}{4-4 \alpha} \varphi(x, 0)
$$

for all $x \in X$.

Proof. Putting $y=0$ in (4), we get

$$
\|2 f(2 x)-4 f(x)\|_{Y} \leq \varphi(x, 0)
$$

for all $x \in X$, since $x \perp 0$. So

$$
\left\|f(x)-\frac{1}{2} f(2 x)\right\|_{Y} \leq \frac{1}{4} \varphi(x, 0)
$$

for all $x \in X$.

Consider the set

$$
S:=\{h: X \rightarrow Y\}
$$

and introduce the generalized metric on $S$ :

$$
d(g, h)=\inf \left\{\mu \in \mathcal{R}_{+}:\|g(x)-h(x)\|_{Y} \leq \mu \varphi(x, 0), \quad \forall x \in X\right\},
$$

where, as usual, inf $\varphi=+\infty$. It is easy to show that $(S, d)$ is complete (see [47]). 
Now we consider the linear mapping $J: S \rightarrow S$ such that

$$
J g(x):=\frac{1}{2} g(2 x)
$$

for all $x \in X$.

Let $g, h \in S$ be given such that $d(g, h)=\varepsilon$. Then

$$
\|g(x)-h(x)\|_{Y} \leq \varphi(x, 0)
$$

for all $x \in X$. Hence

$$
\|J g(x)-J h(x)\|_{Y}=\left\|\frac{1}{2} g(2 x)-\frac{1}{2} h(2 x)\right\|_{Y} \leq \alpha \varphi(x, 0)
$$

for all $x \in X$. So $d(g, h)=\varepsilon$ implies that $d(J g, J h) \leq \alpha \varepsilon$. This means that

$$
d(J g, J h) \leq \alpha d(g, h)
$$

for all $g, h \in S$.

It follows from $(7)$ that $d(f, J f) \leq \frac{1}{4}$.

By Theorem 1.1, there exists a mapping $L: X \rightarrow Y$ satisfying the following:

(1) $L$ is a fixed point of $J$, i.e.,

$$
L(2 x)=2 L(x)
$$

for all $x \in X$. The mapping $L$ is a unique fixed point of $J$ in the set

$$
M=\{g \in S: d(h, g)<\infty\}
$$

This implies that $L$ is a unique mapping satisfying (8) such that there exists a $\mu \in(0$, $\infty)$ satisfying

$$
\|f(x)-L(x)\|_{Y} \leq \mu \varphi(x, 0)
$$

for all $x \in X$;

(2) $d\left(J^{n} f, L\right) \rightarrow 0$ as $n \rightarrow \infty$. This implies the equality

$$
\lim _{n \rightarrow \infty} \frac{1}{2^{n}} f\left(2^{n} x\right)=L(x)
$$

for all $x \in X$;

(3) $d(f, L) \leq \frac{1}{1-\alpha} d(f, J f)$, which implies the inequality

$$
d(f, L) \leq \frac{1}{4-4 \alpha}
$$

This implies that the inequality (5) holds. 
It follows from (3) and (4) that

$$
\begin{aligned}
\|D L(x, y)\|_{Y} & =\lim _{n \rightarrow \infty} \frac{1}{2^{n}}\left\|D f\left(2^{n} x, 2^{n} y\right)\right\|_{Y} \\
& \leq \lim _{n \rightarrow \infty} \frac{1}{2^{n}} \varphi\left(2^{n} x, 2^{n} y\right) \leq \lim _{n \rightarrow \infty} \frac{2^{n} \alpha^{n}}{2^{n}} \varphi(x, y)=0
\end{aligned}
$$

for all $x, y \in X$ with $x \perp y$. So

$$
D L(x, y)=0
$$

for all $x, y \in X$ with $x \perp y$. Since $f$ is odd, $L$ is odd. Hence $L: X \rightarrow Y$ is an orthogonally additive mapping, i.e.,

$$
L(2 x+y)+L(2 x-y)=4 L(x+y)+4 L(x-y)-4 L(x)
$$

for all $x, y \in X$ with $x \perp y$. Thus $L: X \rightarrow Y$ is a unique orthogonally additive mapping satisfying (5), as desired.

From now on, in corollaries, assume that $(X, \perp)$ is an orthogonality normed space.

Corollary 2.3 Let $\theta$ be a positive real number and $p$ a real number with $0<p<1$. Let $f: X \rightarrow Y$ be an odd mapping satisfying

$$
\|D f(x, y)\|_{Y} \leq \theta\left(\|x\|^{p}+\|y\|^{p}\right)
$$

for all $x, y \in X$ with $x \perp y$. Then there exists a unique orthogonally additive mapping $L: X \rightarrow Y$ such that

$$
\|f(x)-L(x)\|_{Y} \leq \frac{\theta}{2\left(2-2^{p}\right)}\|x\|^{p}
$$

for all $x \in X$.

Proof. The proof follows from Theorem 2.2 by taking $\phi(x, y)=\theta\left(\|x\|^{p}+\|y\|^{p}\right)$ for all $x, y \in X$ with $x \perp y$. Then we can choose $\alpha=2^{p-1}$ and we get the desired result.

Theorem 2.4 Let $f: X \rightarrow Y$ be an odd mapping satisfying (4) for which there exists a function $\phi: X^{2} \rightarrow[0, \infty)$ such that

$$
\varphi(x, y) \leq \frac{\alpha}{2} \varphi(2 x, 2 y)
$$

for all $x, y \in X$ with $x \perp y$. Then there exists a unique orthogonally additive mapping $L: X \rightarrow Y$ such that

$$
\|f(x)-L(x)\|_{Y} \leq \frac{\alpha}{4-4 \alpha} \varphi(x, 0)
$$

for all $x \in X$.

Proof. Let $(S, d)$ be the generalized metric space defined in the proof of Theorem 2.2. Now we consider the linear mapping $J: S \rightarrow S$ such that

$$
J g(x):=2 g\left(\frac{x}{2}\right)
$$

for all $x \in X$. 
It follows from (6) that $d(f, J f) \leq \frac{\alpha}{4}$. So

$$
d(f, L) \leq \frac{\alpha}{4-4 \alpha} .
$$

Thus we obtain the inequality (10).

The rest of the proof is similar to the proof of Theorem 2.2.

Corollary 2.5 Let $\theta$ be a positive real number and $p$ a real number with $p>1$. Let $f$ : $X \rightarrow Y$ be an odd mapping satisfying (9). Then there exists a unique orthogonally additive mapping $L: X \rightarrow Y$ such that

$$
\|f(x)-L(x)\|_{Y} \leq \frac{\theta}{2\left(2^{p}-2\right)}\|x\|^{p}
$$

for all $x \in X$.

Proof. The proof follows from Theorem 2.4 by taking $\phi(x, y)=\theta\left(\|x\|^{p}+\|y\|^{p}\right)$ for all $x, y \in X$ with $x \perp y$. Then we can choose $\alpha=2^{1-p}$ and we get the desired result.

\section{Stability of the orthogonally additive-quartic functional equation: an even mapping case}

In this section, applying some ideas from [16,19], we deal with the stability problem for the orthogonally additive-quartic functional equation given in the previous section: an even mapping case.

Definition 3.1 A mapping $f: X \rightarrow Y$ is called an orthogonally quartic mapping if

$$
f(2 x+y)+f(2 x-y)=4 f(x+y)+4 f(x-y)+24 f(x)-6 f(y)
$$

for all $x, y \in X$ with $x \perp y$.

Theorem 3.2 Let $\phi: X^{2} \rightarrow[0, \infty)$ be a function such that there exists an $\alpha<1$ with

$$
\varphi(x, y) \leq 16 \alpha \varphi\left(\frac{x}{2}, \frac{y}{2}\right)
$$

for all $x, y \in X$ with $x \perp y$. Let $f: X \rightarrow Y$ be an even mapping satisfying $f(0)=0$ and (4). Then there exists a unique orthogonally quartic mapping $P: X \rightarrow Y$ such that

$$
\|f(x)-P(x)\|_{Y} \leq \frac{1}{32-32 \alpha} \varphi(x, 0)
$$

for all $x \in X$.

Proof. Putting $y=0$ in (4), we get

$$
\|2 f(2 x)-32 f(x)\|_{Y} \leq \varphi(x, 0)
$$

for all $x \in X$, since $x \perp 0$. So

$$
\left\|f(x)-\frac{1}{16} f(2 x)\right\|_{Y} \leq \frac{1}{32} \varphi(x, 0)
$$

for all $x \in X$.

Let $(S, d)$ be the generalized metric space defined in the proof of Theorem 2.2. Now we consider the linear mapping $J: S \rightarrow S$ such that 


$$
J g(x):=\frac{1}{16} g(2 x)
$$

for all $x \in X$.

The rest of the proof is similar to the proof of Theorem 2.2.

Corollary 3.3 Let $\theta$ be a positive real number and $p$ a real number with $0<p<4$. Let $f: X \rightarrow Y$ be an even mapping satisfying $f(0)=0$ and (9). Then there exists a unique orthogonally quartic mapping $P: X \rightarrow Y$ such that

$$
\|f(x)-P(x)\|_{Y} \leq \frac{\theta}{2\left(16-2^{p}\right)}\|x\|^{p}
$$

for all $x \in X$.

Proof. The proof follows from Theorem 3.2 by taking $\phi(x, y)=\theta\left(\|x\|^{p}+\|y\|^{p}\right)$ for all $x, y \in X$ with $x \perp y$. Then we can choose $\alpha=2^{p-4}$ and we get the desired result.

Theorem 3.4 Let $f: X \rightarrow Y$ be an even mapping satisfying (4) and $f(0)=0$ for which there exists a function $\phi: X^{2} \rightarrow[0, \infty)$ such that

$$
\varphi(x, y) \leq \frac{\alpha}{16} \varphi(2 x, 2 y)
$$

for all $x, y \in X$ with $x \perp y$. There exists a unique orthogonally quartic mapping $P: X \rightarrow Y$ such that

$$
\|f(x)-P(x)\|_{Y} \leq \frac{\alpha}{32-32 \alpha} \varphi(x, 0)
$$

for all $x \in X$

Proof. Let $(S, d)$ be the generalized metric space defined in the proof of Theorem 2.2.

Now we consider the linear mapping $J: S \rightarrow S$ such that

$$
J g(x):=16 g\left(\frac{x}{2}\right)
$$

for all $x \in X$.

It follows from (11) that $d(f, J f) \leq \frac{\alpha}{32}$. So we obtain the inequality (12).

The rest of the proof is similar to the proof of Theorem 2.2.

Corollary 3.5 Let $\theta$ be a positive real number and $p$ a real number with $p>4$. Let $f$ : $X \rightarrow Y$ be an even mapping satisfying $f(0)=0$ and (9). Then there exists a unique orthogonally quartic mapping $P: X \rightarrow Y$ such that

$$
\|f(x)-P(x)\|_{Y} \leq \frac{\theta}{2\left(2^{p}-16\right)}\|x\|^{p}
$$

for all $x \in X$.

Proof. The proof follows from Theorem 3.4 by taking $\phi(x, y)=\theta\left(\|x\|^{p}+\|y\|^{p}\right)$ for all $x, y \in X$ with $x \perp y$. Then we can choose $\alpha=2^{4-p}$ and we get the desired result.

$$
\text { Let } f_{o}(x)=\frac{f(x)-f(-x)}{2} \text { and } f_{e}(x)=\frac{f(x)+f(-x)}{2} \text {. Then } f_{o} \text { is an odd mapping and } f_{e}
$$
is an even mapping such that $f=f_{o}+f_{e}$.

The above corollaries can be summarized as follows: 
Theorem 3.6 Assume that $(X, \perp)$ is an orthogonality normed space. Let $\theta$ be a positive real number and $p$ a real number with $0<p<1$ or $p>4$. Let $f: X \rightarrow Y$ be a mapping satisfying $f(0)=0$ and (9). Then there exist an orthogonally additive mapping $L$ : $X \rightarrow Y$ and an orthogonally quartic mapping $P: X \rightarrow Y$ such that

$$
\|f(x)-L(x)-P(x)\|_{Y} \leq\left(\frac{1}{\left|2-2^{p}\right|}+\frac{1}{\left|16-2^{p}\right|}\right) \frac{\theta}{2}\|x\|^{p}
$$

for all $x \in X$.

\section{Acknowledgements}

This work was supported by the Daejin University Research Grants in 2012.

\section{Author details}

${ }^{1}$ Department of Mathematics, Daejin University, Kyeonggi 487-711, Korea ${ }^{2}$ Department of Mathematics, Research Institute for Natural Sciences, Hanyang University, Seoul 133-791, South Korea ${ }^{3}$ Department of Mathematics and Computer Science, Iran University of Science and Technology, Behshahr, Iran

\section{Authors' contributions}

All authors carried out the proof. All authors conceived of the study, and participated in its design and coordination. All authors read and approved the final manuscript.

\section{Competing interests}

The authors declare that they have no competing interests.

Received: 22 April 2011 Accepted: 12 April 2012 Published: 12 April 2012

\section{References}

1. Pinsker, AG: Sur une fonctionnelle dans l'espace de Hilbert. C R (Dokl) Acad Sci URSS, n Ser. 20, 411-414 (1938)

2. Sundaresan, K: Orthogonality and nonlinear functionals on Banach spaces. Proc Am Math Soc. 34, 187-190 (1972)

3. Gudder, S, Strawther, D: Orthogonally additive and orthogonally increasing functions on vector spaces. Pac J Math. 58, 427-436 (1975)

4. Rätz, J: On orthogonally additive mappings. Aequationes Math. 28, 35-49 (1985)

5. Rätz, J, Szabó, Gy: On orthogonally additive mappings IV. Aequationes Math. 38, 73-85 (1989)

6. Alonso, J, Benítez, C: Orthogonality in normed linear spaces: a survey I. Main properties Extracta Math. 3, 1-15 (1988)

7. Alonso, J, Benítez, C: Orthogonality in normed linear spaces: a survey II. Relations between main orthogonalities Extracta Math. 4, 121-131 (1989)

8. Birkhoff, G: Orthogonality in linear metric spaces. Duke Math J. 1, 169-172 (1935)

9. Carlsson, SO: Orthogonality in normed linear spaces. Ark Mat. 4, 297-318 (1962)

10. Diminnie, CR: A new orthogonality relation for normed linear spaces. Math Nachr. 114, 197-203 (1983)

11. James, RC: Orthogonality and linear functionals in normed linear spaces. Trans Am Math Soc. 61, 265-292 (1947)

12. Ulam, SM: Problems in Modern Mathematics. Wiley, New York (1960)

13. Hyers, DH: On the stability of the linear functional equation. Proc Nat Acad Sci USA. 27, 222-224 (1941)

14. Rassias, ThM: On the stability of the linear mapping in Banach spaces. Proc Amer Math Soc. 72, $297-300$ (1978)

15. Czerwik, S: Stability of Functional Equations of Ulam-Hyers-Rassias Type. Hadronic Press, Palm Harbor, Florida. (2003)

16. Hyers, DH, Isac, G, Rassias, ThM: Stability of Functional Equations in Several Variables. Birkhäuser, Basel (1998)

17. Jung, S: Hyers-Ulam-Rassias Stability of Functional Equations in Mathematical Analysis. Hadronic Press, Palm Harbor, Florida (2001)

18. Rassias, ThM: Functional Equations, Inequalities and Applications. Kluwer Academic Publishers, Dordrecht/Boston/ London (2003)

19. Ger, R, Sikorska, J: Stability of the orthogonal additivity. Bull Polish Acad Sci Math. 43, 143-151 (1995)

20. Skof, F: Proprietà locali e approssimazione di operatori. Rend Sem Mat Fis Milano. 53, 113-129 (1983)

21. Cholewa, PW: Remarks on the stability of functional equations. Aequationes Math. 27, 76-86 (1984)

22. Czerwik, S: On the stability of the quadratic mapping in normed spaces. Abh Math Sem Univ Hamburg. 62, 59-64 (1992)

23. Czerwik, S: Functional Equations and Inequalities in Several Variables. World Scientific Publishing Company/New Jersey/ London/Singapore/Hong Kong (2002)

24. Park, C, Park, J: Generalized Hyers-Ulam stability of an Euler-Lagrange type additive mapping. J Diff Equ Appl. 12, 1277-1288 (2006)

25. Rassias, ThM: On the stability of the quadratic functional equation and its applications. Studia Univ Babeş-Bolyai Math. 43, 89-124 (1998)

26. Rassias, ThM: The problem of S.M. Ulam for approximately multiplicative mappings. J Math Anal Appl. 246, 352-378 (2000)

27. Rassias, ThM: On the stability of functional equations in Banach spaces. J Math Anal Appl. 251, 264-284 (2000)

28. Vajzović, F: Über das Funktional $H$ mit der Eigenschaft: $(x, y)=0 \otimes H(x+y)+H(x-y)=2 H(x)+2 H(y)$. Glasnik Mat Ser II. 2(22):73-81 (1967)

29. Drljević, F: On a functional which is quadratic on A-orthogonal vectors. Publ Inst Math (Beograd). 54, 63-71 (1986) 
30. Fochi, M: Functional equations in A-orthogonal vectors. Aequationes Math. 38, $28-40$ (1989)

31. Moslehian, MS: On the orthogonal stability of the Pexiderized quadratic equation. J Diff Equ Appl. 11, 999-1004 (2005)

32. Moslehian, MS: On the stability of the orthogonal Pexiderized Cauchy equation. J Math Anal Appl. 318, 211-223 (2006)

33. Szabó, Gy: Sesquilinear-orthogonally quadratic mappings. Aequationes Math. 40, 190-200 (1990)

34. Moslehian, MS, Rassias, ThM: Orthogonal stability of additive type equations. Aequa-tiones Math. 73, 249-259 (2007)

35. Paganoni, L, Rätz, J: Conditional function equations and orthogonal additivity. Aequa-tiones Math. 50, 135-142 (1995)

36. Cădariu, L, Radu, V: Fixed points and the stability of Jensen's functional equation. J Inequal Pure Appl Math 4(1):7 (2003). (Art. ID 4)

37. Diaz, J, Margolis, B: A fixed point theorem of the alternative for contractions on a generalized complete metric space. Bull Am Math Soc. 74, 305-309 (1968)

38. Isac, G, Rassias, ThM: Stability of $\psi$-additive mappings: appications to nonlinear analysis. Int J Math Math Sci. 19, 219-228 (1996)

39. Cădariu, L, Radu, V: On the stability of the Cauchy functional equation: a fixed point approach. Grazer Math Ber. 346, 43-52 (2004)

40. Cădariu, L, Radu, V: Fixed point methods for the generalized stability of functional equations in a single variable. Fixed Point Theory Appl 2008, 1-5 (2008). (Art. ID 749392)

41. Jung, Y, Chang, I: The stability of a cubic type functional equation with the fixed point alternative. J Math Anal Appl. 306, 752-760 (2005)

42. Mirzavaziri, M, Moslehian, MS: A fixed point approach to stability of a quadratic equation. Bull Braz Math Soc. 37, 361-376 (2006)

43. Saadati, R, Park, C: Non-Archimedean $\bigotimes$-fuzzy normed spaces and stability of functional equations. Comput Math Appl. 60, 2488-2496 (2010)

44. Saadati, R, Vaezpour, SM, Park, C: The stability of the cubic functional equation in various spaces. Math Commun. 16, $131-145$ (2011)

45. Radu, V: The fixed point alternative and the stability of functional equations. Fixed Point Theory. 4, 91-96 (2003)

46. Lee, S, Im, S, Hwang, I: Quartic functional equations. J Math Anal Appl. 307, 387-394 (2005)

47. Mihet, D, Radu, V: On the stability of the additive Cauchy functional equation in random normed spaces. J Math Anal Appl. 343, 567-572 (2008)

doi:10.1186/1029-242X-2012-83

Cite this article as: Lee et al: Orthogonal stability of an additive-quartic functional equation with the fixed point alternative. Journal of Inequalities and Applications 2012 2012:83.

\section{Submit your manuscript to a SpringerOpen ${ }^{\circ}$} journal and benefit from:

- Convenient online submission

- Rigorous peer review

- Immediate publication on acceptance

- Open access: articles freely available online

- High visibility within the field

- Retaining the copyright to your article

Submit your next manuscript at $\gg$ springeropen.com 